\title{
Transformation of the Physics and Astronomy courses
}

\author{
Dmitriy Beznosko ${ }^{a}{ }^{*}$, Tatiana Krivosheev ${ }^{a}$ and Alexander lakovlev $\boldsymbol{b}$ \\ a Clayton State University, \\ 2000 Clayton State Blvd., Morrow GA 30260, USA \\ b Computer Systems Institute, \\ 529 Main Street, Charlestown MA 02129, USA \\ E-mail: dmitriybeznosko@clayton.edu
}

The science education in universities has several deferring moments for the general student population outside of the Physics/Astronomy fields or STEM in general. A large contribution to that comes from the high cost of the textbooks that is typical for the introductory physics and astronomy courses. Another is the lack of supporting class materials, such as audio-video materials and support tools for activities. This poster will cover the class transformation activities under the ALG grant to adopt the free textbooks from OpenStax for the intro Physics 1-2 and Astronomy 12 sequences, and to create the supporting materials such as presentations, tests, audio-video materials, and in-browser run online tools for the class activities as applicable to the courses listed above. Adaptations to online or hybrid teaching style will be also noted, and students' survey results will be included as well. 


\section{Introduction}

At the majority of the educational institutions, a typical science textbook that is used for the introductory science classes at a freshman level, such as physics or astronomy and similar, can run to a heavy $\$ 150$ - $\$ 250$ range. This additional expense to the rising overall cost of the education can be a deferring factor that increases the financial burden on a student and could lead to the student choosing a similar course with lower expenses. This effect has become more pronounced in the current economic situation and at the primarily minority-oriented educational institutions.

Today, there are textbooks for many popular subjects that are available online for free (with typically a small charge for the printed version). The adoption of a new textbook for a large course such as the Introductory Physics or Introductory Astronomy is an extensive undertaking with the need to create also the class materials, quiz and exam test banks, homework assignments as well as additional collaborative projects and online tools for the remote or hybrid course delivery.

There are different programs supporting the transformation of the courses towards reducing the financial burden on students by switching to Open Educational Resources (OER) available and also creating the needed 'ecosystem' around the new adopted materials and textbooks. The transformations of the intro Astronomy and Physics courses mentioned in this publication were supported by ALG (Affordable Learning Georgia) [1].

\section{An Open Textbook for Science Course}

While there are few low-cost and/or free textbooks available, the rising popularity is for the OpenStax [2] collection of the textbooks. Their textbooks for the Astronomy (for non-science majors), for Introductory Physics (with calculus) and the supporting OER were adopted as part of this work.

There are certain Pros and Cons in using a textbook similar to described above. The Pros are the free download/online access, very affordable printed copy that few students do prefer, typically a community driven collection of OER and a wide variety of formats for downloads. With that come the Cons such as simple-looking design, very limited collection/banks of test questions and usually no ready slides that could be adopted (for example, the OpenStax slides are just the figures from the textbook with caption). Also, as with any new textbook, the order of subjects changes and necessitates the changes not only in quiz/exam schedule but also to the order of the accompanying labs.

\subsection{Survey Results on Textbook}

At the end of the Spring 2021 semester the survey was done in the Astronomy course that ran with the OpenStax textbook for the first time during that semester to gauge students' reaction to the new textbook.

The first question asked was to see if, due to the textbook choice, students were more likely to register for the course and if their overall financial obligations as students were noticeably reduces. Figure 1a demonstrates that a majority of students strongly agreed with that.

Another question was also asked about the design of the new textbook. The survey result in Figure $1 \mathrm{~b}$ shows that most students did like the simpler design, and everyone say that it is about 
the same or better than the usual textbooks in similar courses. Only two students noted that additional resources available are somewhat worse than for non-free books.

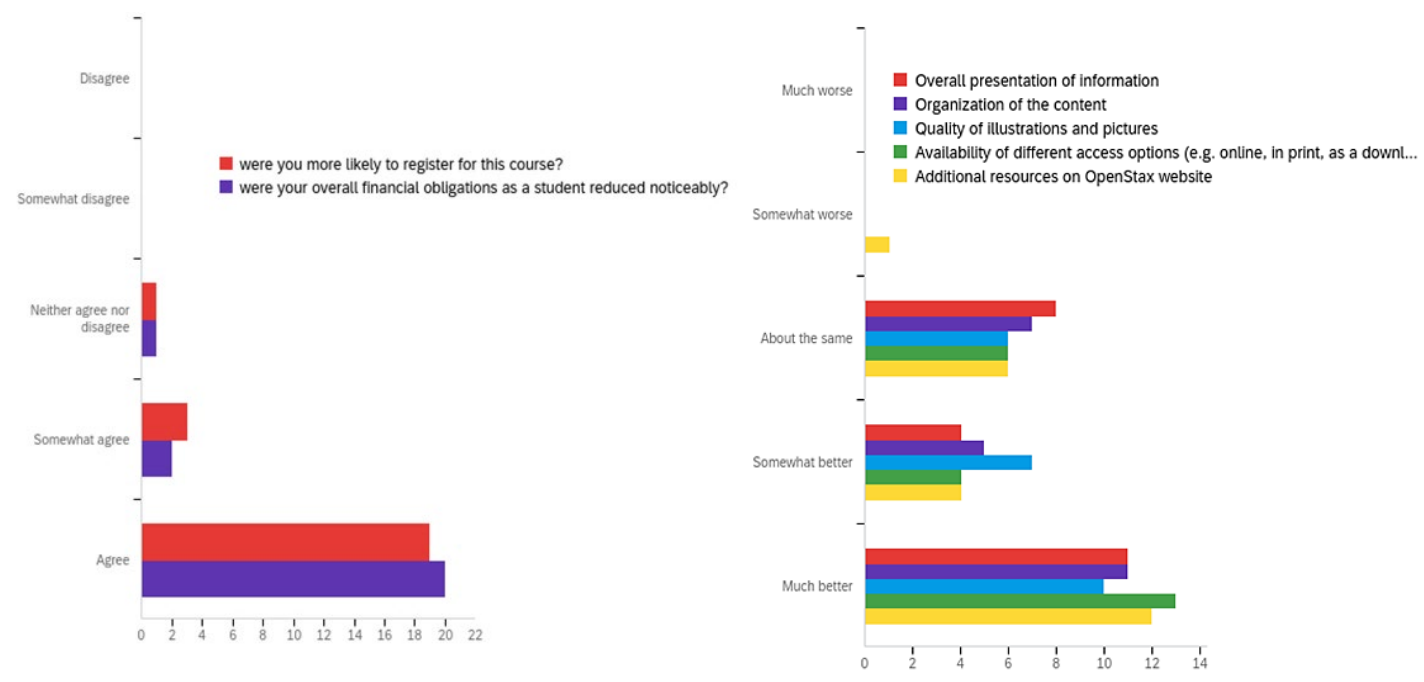

Figure 1: Survey result to the question about the textbook: $a$-impact onto students, $b$ - design.

Another part of the survey asked how students accessed the textbook (Figure 2a) and whether the cost would be a deterring factor for registering for the Astronomy course (Figure 2b). Only a small fraction of students did purchase a printed copy, all others preferred the online access via personal computer or tablet, and/or downloading the provided pdf file. As for the cost of the textbook, most students agreed that other financial obligations during a semester would be the main driving factor whether this additional expense will serve as a deterring factor.
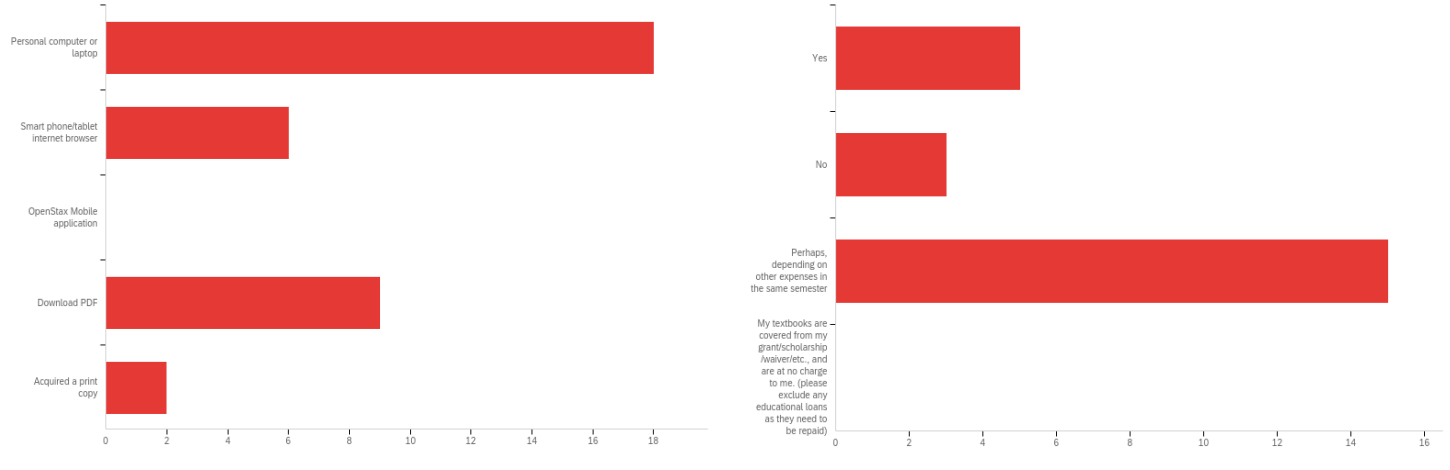

Figure 2: Survey result to the question about: $a$-access to textbook, $b$-cost being a deterrent.

\section{Online Tools}

As part of this project, three online tools have been developed with at least another three planned until the end of the project. These tools are currently for Astronomy course only, with the planned ones to extend the Physics course capabilities as well. All tools are under the CC BY (creative commons attribution license) and could be used by anyone - available at: https://github.com/aiakovlev/ASTR-1010-1020. 
The current tools are: the Solar rotation tool, the online reporting tool and the Moon observation logger tool. All are written in JavaScript and executed in the web browser without any additional libraries or modules that need to be installed on the user computer. This is especially useful if a student uses university computer or accesses the tool from a phone or tablet.

The Solar rotation tool gives students the ability to visually analyze a motion of sunspots across the disk of the Sun to measure its rotation period at the latitude of the sunspots. Students are asked to download a series of at least 5 to 7 images with the same spots traceable and use them in the program to obtain the results as a plot of position vs time, and the period of rotation from the slope of the fit line done automatically (Figure $3 a$ and Figure $3 b$ ).

The online reporting tool allows students to create a report on any object in the Solar system by pretending to be a secret agent of an alien species. While the tool provides some animation and encoding into 'alien' language as part of the interactive abilities, it is a simple text editing tool with the capabilities to insert images and to produce an output file in the pdf format for submission. This is all done in the web browser without any need for the word processing software and can be used on the smartphone and the tablet as well (Figure 3c).
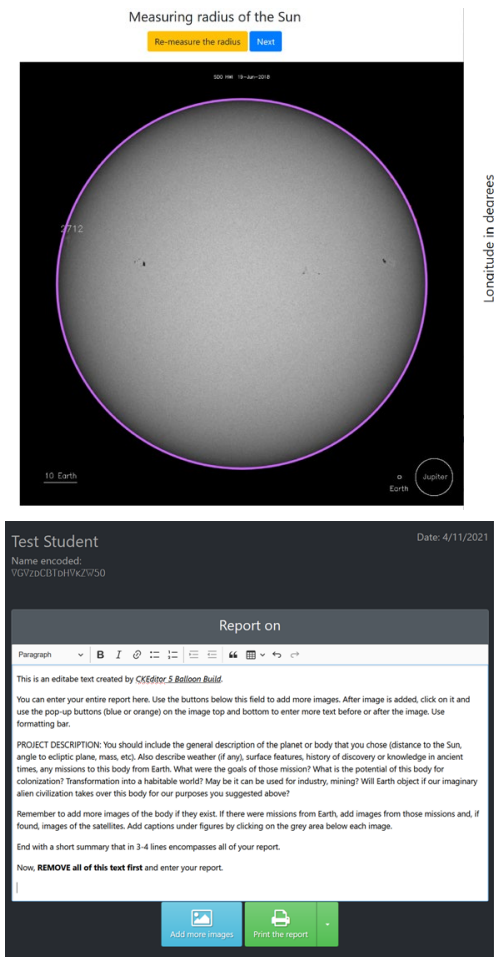

Figure 3: Online tool screenshots:

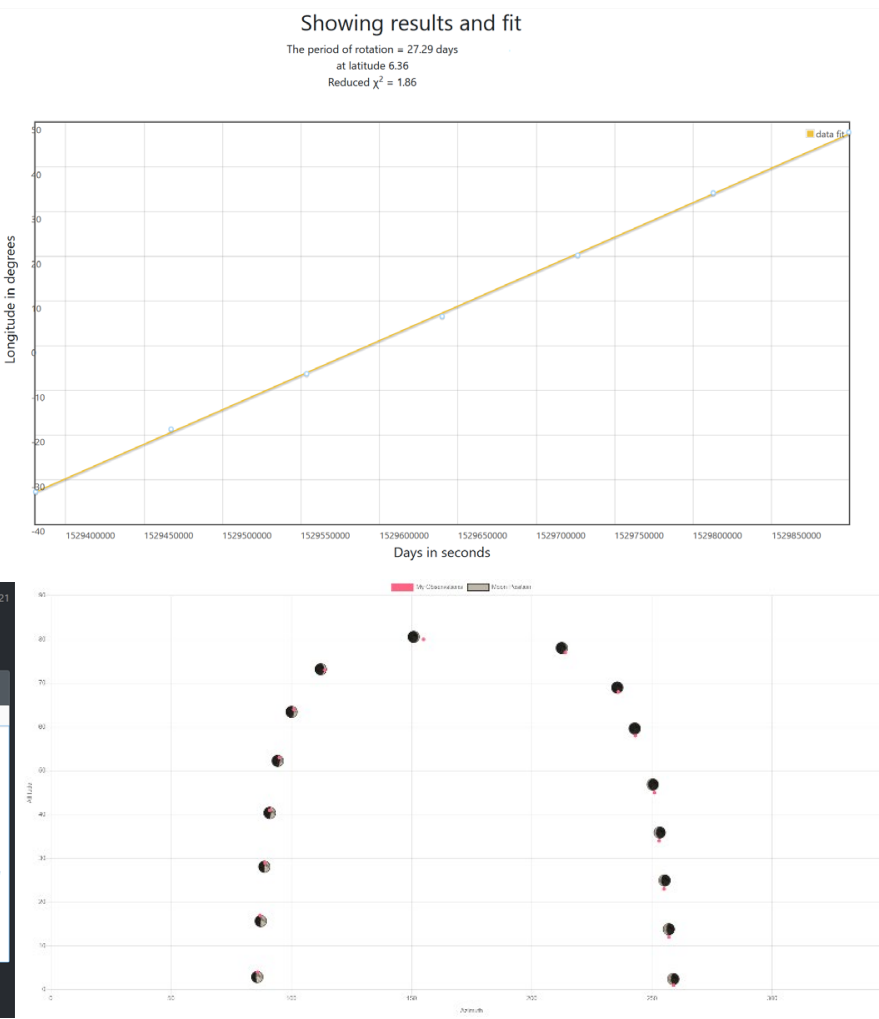

$a$ and $b$-Solar rotation tool, $c$-Online reporting tool and $d-$ Moon observation data logging tool.

The last of the current tools is the Moon observation and data logger tool. This tool was written after students answered in the survey that it would be very useful for them during the Moon observation project (during the semester, this project was assigned first before any tools were written). It allows students to log the observational data (elevation and the azimuth) in the web browser and makes the plot with red dots being the student data superimposed on the actual position and phase of the Moon on the time and date of the observations. 
The survey shows that overwhelming majority of students has liked the online tools with only exception of a few who couldn't run them for various reasons (such as missing a class with explanation on how to use the tools and not coming to office hours as well) and had to use the backup options, such as using the word processing and spreadsheet software.

\section{Laboratory Adjustment Preliminaries}

In the Spring 2020, we had to reconsider the ways we teach physics laboratories due to sudden switch to remote methods of course delivery due to Covid-19 pandemics. In the attempts to deliver meaningful experience during a stay-home lockdown and preserve the integrity of the course, we decided to deliver the laboratory experiments in Mechanics using the Tracker [3] software for physical simulation and video analysis.

Students were given step by step instructions on how to set up a video of a physical system using some everyday objects easily found around home, and then perform video calibration and data collecting in Tracker. Students were given an option of first making their own video, or use one of the several videos provided by an instructor. Although a significant number of students were using their own videos, an instructor provided videos were popular since it was sometimes difficult for a student to provide necessary everyday objects needed for an experiment. In particular, we discovered that students sometimes did not have measuring devices such as rulers or meter sticks for a video calibration. Even rarer were rubber balls and balances available. This difficulty could be resolved to some degree by using objects of well-known dimensions (such as copy paper) and/or masses (coins). Once a video was calibrated, the Tracker provided measurements of an object (or objects) positions with a great degree of precision and accuracy. In fact, we found that all the labs that were quickly converted to the video analysis format produced as good or (in most cases) better results compared to the experiments performed by students in the laboratory setting. Among the laboratories originally converted to the video analysis format were Conservation of Energy (in Atwood machine configuration), Rolling Motion, Collisions, and Forces of Friction.

The following Fall 2020 semester brought new challenges to teaching the laboratories, since the Covid-19 limitations required us to reduce the number of students present in the lab simultaneously to one half of the usual amount. We made the decision of teaching the mechanics laboratories in hybrid format - in which a student would come to the lab once every two weeks for a traditional experiment while performing in-home Tracker based experiment on alternative weeks. Another laboratory, Projectile Motion, was developed fast to complement the existing assortment and to give the overall 6 home-based experiments to students.

Overall, the preliminary Tracker experiments were well received by the students. The common comments on the exit surveys shown in Figure 4 were "Tracker was easy/convenient/powerful to use", "convenient to perform experiments at your own pace/time", "well supplemented by manual/instructions", "can figure out details in depth".

Students commented on the convenience of having the step-by-step instructions in a video format, since they could always go back and watch parts again. Interestingly enough, students found home-based labs easier to perform compared to the laboratory-based experiments. While asked if home based labs helped them to get comfortable with new technology tools, most students 
responded that they have gotten very comfortable not only with the video analysis tools, but also using Excel to process the data.

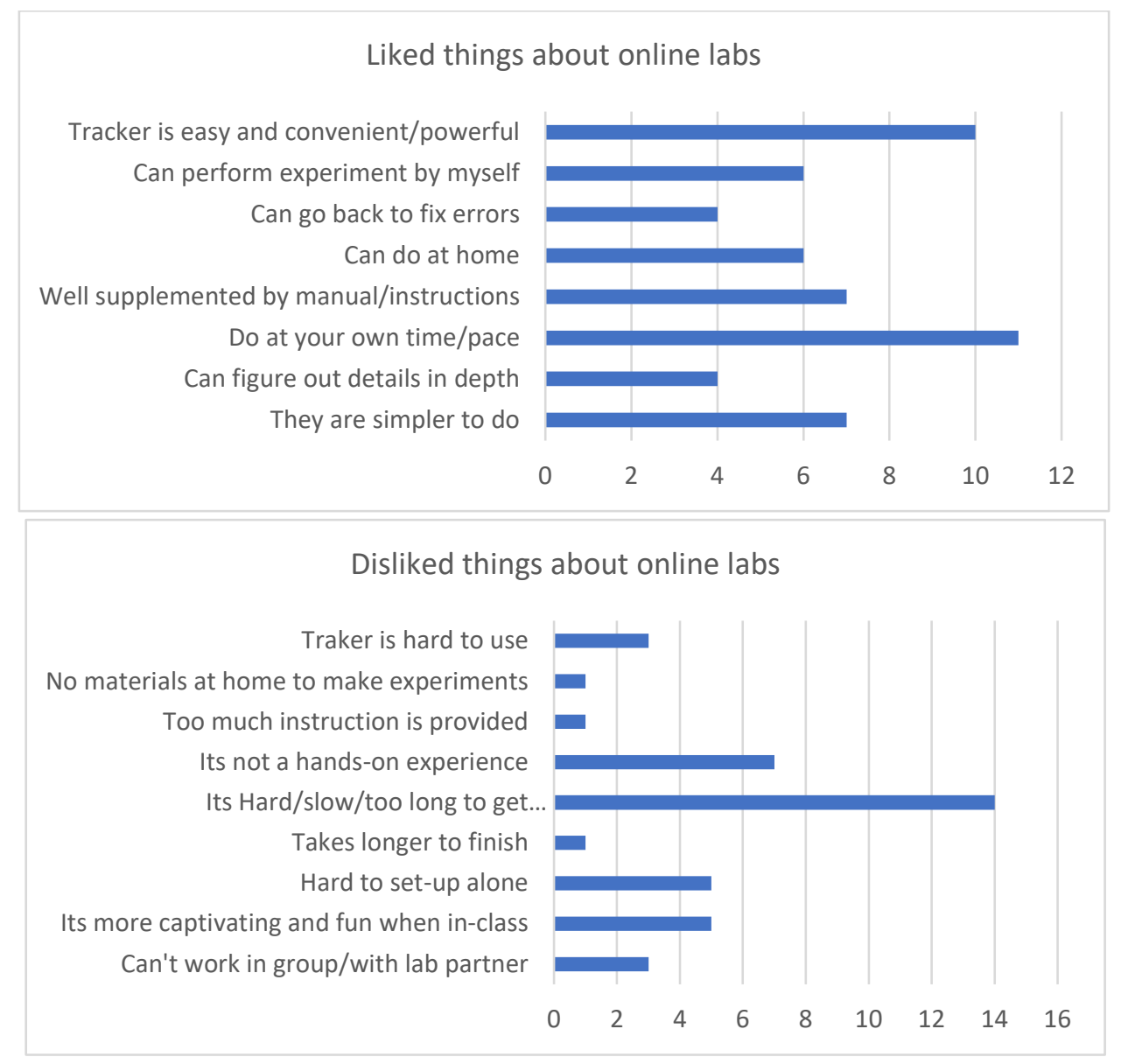

Figure 4: Survey result about the online laboratories.

Early on we recognized that one element of a successful lab experience, that is a partner corroboration and mutual learning, is difficult to fully recreate in case of the home-based experiments. We attempted to compensate for it by offering our students an opportunity to create "group" lab reports rather than individual ones in hopes to booster collaboration. Interestingly enough, some students who worked on their own laboratory experiments and lab reports, most commonly cited difficulty in finding time convenient for everybody as a reason.

Using Tracker based labs allowed us to significantly expand the range of the topics that we can offer in the laboratory without investing in expensive apparatus. We plan to use this idea and gained experience to apply for the ALG 'course transformation' grant so that the videos of the experiments will be re-done in higher quality, more experiments will be available for choosing and the lab manuals will be updated specifically for the use of the Tracker for these experiments.

\section{Conclusion}

The experience of the past semesters for the laboratories and the last semester with the adaptation of the new textbook and the online tools has provided us with invaluable experience. We know that students agree that the free textbook is overall better in their opinion than other 
textbooks from their experience as a student. This will allow us to proceed with converting other courses to a free textbook. With that move, the overall financial burden on the students will be significantly reduced. The online reporting tools are generally liked (with very few students reporting technical difficulties).

The preliminary attempts with the online lab experiments are much preferred over the usual in-class laboratories, and we will continue expanding this idea with a goal to introduce up to $50 \%$ of all lab experiments as 'online' in the future.

\section{Acknowledgement}

This work was sponsored in part by the Affordable Materials grant \#561 provided by Board of Regents of the university System of Georgia.

\section{References}

[1] University System of Georgia, Affordable Learning Georgia, https://www.affordablelearninggeorgia.org/ 2021

[2] RICE University, Open Stax project, https://openstax.org/2021

[3] Douglas Brown, Wolfgang Christian, Robert M Hanson, Tracker Video Analysis and Modeling Tool, https://physlets.org/tracker/ 2021 